\title{
Photoluminescence and structural properties of zirconium dioxide thin films produced by RF sputtering technique
}

\author{
Pinar K. Bakacak ${ }^{1}$, Emre Gur², Ozkan Bayram ${ }^{3, *}$ (D), Sebahattin Tuzemen², and \\ Onder Simsek ${ }^{4,5}$ \\ ${ }^{1}$ Department of Physics, Faculty of Science, Bilkent University, 06100 Ankara, Turkey \\ ${ }^{2}$ Department of Physics, Faculty of Science, Atatürk University, 25240 Erzurum, Turkey \\ ${ }^{3}$ Electrical-Electronics Engineering, Engineering Faculty, Bayburt University, 69100 Bayburt, Turkey \\ ${ }^{4}$ Department of Electric and Energy, Vocational School, Agri Ibrahim Cecen University, 04100 Agri, Turkey \\ ${ }^{5}$ Central Application and Research Laboratory (MERLAB), Agri Ibrahim Cecen University, 04100 Agri, Turkey
}

Received: 9 August 2020

Accepted: 3 February 2021

Published online:

3 March 2021

(C) The Author(s), under exclusive licence to Springer Science + Business Media, LLC part of Springer Nature 2021

\begin{abstract}
In this study, Zirconium Oxide $\left(\mathrm{ZrO}_{2}\right)$ thin films were produced by using radio frequency magnetron sputtering method on glass substrate at various pressures. The effects of growth pressure on the characteristics of grown Zirconia nanostructures were investigated by Atomic Force Microscopy (AFM), Scanning Electron Microscopy (SEM), X-Ray Diffraction (XRD), Uv-Visible Spectroscopy and Photoluminescence Spectrometry (PL). XRD analysis showed that peaks of the monoclinic and tetragonal phases were more effective at low growth pressure, but different monoclinic phase peaks were present at increasing growth pressures. The surface morphological properties of the films such as average and maximum roughness, changed significantly with increasing growth pressure, and the maximum peak height first increased to $40 \mathrm{~nm}$ and then decreased to $20 \mathrm{~nm}$. The optical transmittance of these thin films was examined in the 450-1100 nm wavelength range, covering both the visible and near-infrared spectral range, and it was found to be approximately $95 \%$ at 550 $\mathrm{nm}$ wavelength, and the optical band gaps were calculated to be approximately $3.26 \mathrm{eV}$ by PL analyses. Uv-Visible spectroscopy analysis showed that the refractive index of the Zirconia films decreases from 2.09 to 1.79 with increasing wavelength of the incident photon. $\mathrm{ZrO}_{2}$ thin films are considered potential materials for transparent electronic devices because of their high transmittance value.
\end{abstract}

Address correspondence to E-mail: obayram@bayburt.edu.tr 


\section{Introduction}

Recently, Zirconium oxide (Zirconia, $\mathrm{ZrO}_{2}$ ) thin films, which are one of the important materials in terms of electrical, optical, and mechanical properties, are getting more and more attention due to their superior properties such as high melting temperature $2680{ }^{\circ} \mathrm{C}$ [1], high resistance to oxidation [2], low thermal conductance at room temperature [3] (4.2 $\mathrm{Wm}^{-1} \mathrm{~K}^{-1}$ at room temperature), and high refractive index [4-6]. $\mathrm{ZrO}_{2}$ is preferred in many important applications such as oxygen gas sensors [7], compact disc (CD) read-heads [8], as a component of solid oxide fuel cells [9], biological ceramics [10], and memory devices [11] due to its excellent properties. $\mathrm{ZrO}_{2}$, one of the transition metal oxides, has a wide band gap from 5.2 to $5.79 \mathrm{eV}$. Some researchers have also reported that these thin films have optically high transparency. The high optical transparency (higher than 95\% within the wavelength range of $450-1100 \mathrm{~nm}$ ) increases the potential of zirconium oxide films to be used as alternative products in device applications. Also, zirconia, which has a high dielectric constant, is accepted as an alternative to $\mathrm{SiO}_{2}$, which is widely used as a dielectric gate. The various crystal structure forms of the zirconium play an important role on the dielectric properties of the material. These crystal structures are monoclinic, cubic, and tetragonal, and the dielectric constant of these phases is known as 20,37 , and 47 , respectively [12]. Furthermore, the dielectric constant (K) for the amorphous crystal phase of zirconium is $\mathrm{K} \sim 22$. Silicon dioxide $\left(\mathrm{SiO}_{2}\right)$, which is used as dielectric gate in metal-oxide-semiconductor (MOS) devices, has high tunnel leakage current and consequently causes device reliability problem. $\mathrm{ZrO}_{2}$ with low leakage current level in the metal-oxide-semiconductor transistors is considered as a potential alternative to a $\mathrm{SiO}_{2}$ gate dielectric below $2 \mathrm{~nm}$ scale [12]. Various physical and chemical growth techniques, such as pulsed ion-beam evaporation [13], sol-gel, chemical vapor deposition [14, 15], successive ionic layer adsorption and reaction technique [16-19], and radio frequency (RF) magnetron sputtering [20-23], are widely used in the production of high quality these oxides. The RF magnetron sputtering technique, which is one of these methods, is more prominent among other techniques because it allows the adjustment of plasma gas ratio $\left(\mathrm{O}_{2} / \mathrm{Ar}+\mathrm{O}_{2}\right)$, substrate temperature, and RF power. In this study, it was aimed to determine the structural, morphological, chemical composition, and optical properties of Zirconia thin films grown using RF magnetron sputtering technique at various growth pressures. AFM, SEM, XRD, Uv-Visible Spectroscopy, PL, and EDAX analyses were performed to characterize thin films and the results of these analyses were compared in detail with the literature studies.

\section{Materials and methods}

The Zirconium ( $\mathrm{Zr}$ ) target material, Argon (Ar) gas and Oxygen $\left(\mathrm{O}_{2}\right)$ gas, which had a purity of $99.99 \%$, was used as the initiator material and gas to obtain $\mathrm{ZrO}_{2}$ thin films. These films were produced on glass substrates using the RF magnetron scattering technique, taking into account coating parameters such as various Ar gas flow rates, $5 \mathrm{~h}$ of coating time, $420{ }^{\circ} \mathrm{C}$ coating temperature, and $80 \mathrm{~W}$ RF power. Before the coating process, the glass substrates were immersed in acetone and methanol for 2 minutes, respectively, and the substrates were rinsed in deionized (DI) water between both processes. Following this process, the substrates were dipped into the piranha $\left(\mathrm{H}_{2} \mathrm{O}_{2}: \mathrm{H}_{2} \mathrm{SO}_{4}(3: 1)\right)$ solution for 1 day. Finally, the substrates were rinsed with DI water and dried with nitrogen $\left(\mathrm{N}_{2}\right)$ gas to make them ready for the growth process. The $\mathrm{Zr}$ target material was plasma treated immediately prior to plating with an $\mathrm{Ar}^{+}$ion plasma to remove possible oxide layer and contamination at these target surfaces. In this study, the gas pressure was increased by keeping the oxygen gas flow constant at $0.75 \mathrm{sccm}$ and gradually changing the argon pressure. Zirconium films deposited using 5, 10, 15, and $20 \mathrm{sccm}$ Ar partially pressure were named S1, S2, S3, and S4, respectively.

\section{Results and discussion}

\subsection{X-ray diffraction studies}

The crystalline structure of the films was examined by X-ray diffraction (XRD) analysis, which has a wavelength of $1.5409 \AA$. XRD measurements were made to investigate the effect of coating pressure on film quality and the results were presented in Fig. 1. These figure shows the XRD models of $\mathrm{ZrO}_{2}$ 


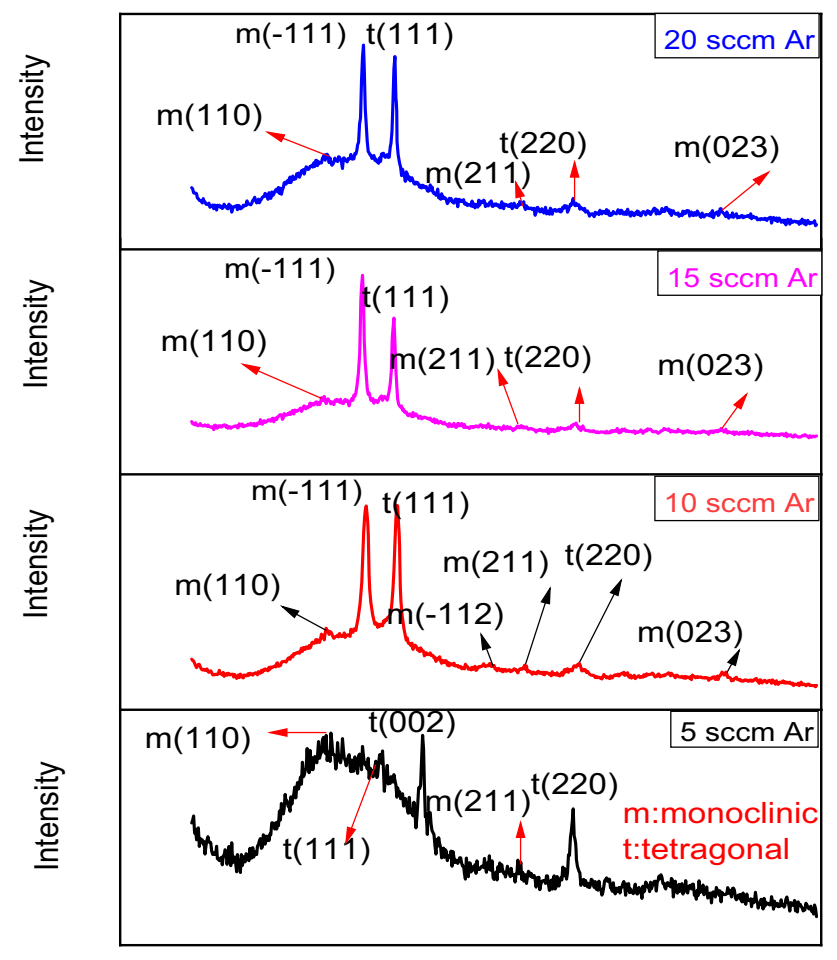

$2 \theta$

Fig. 1 XRD graphs of Zirconium nanostructures produced using various Ar gas flow rates

nanostructured films grown at different Ar gas flow rates. XRD patterns showed a significant effect of gas pressure on the crystal structure of the films. For the deposited films, the observed reflections at $2 \theta=24.6$, $2 \theta=28.2^{\circ}, 2 \theta=44^{\circ}$, and $2 \theta=66^{\circ}$ were related to the $\mathrm{m}$ (110), $\mathrm{m}(-111), \mathrm{m}(211)$, and $\mathrm{m}(023)$ planes of monoclinic phase ( $\mathrm{m}-\mathrm{ZrO}_{2}$, JCPDS card No. 88-2390) and $2 \theta=31^{\circ}, 2 \theta=50^{\circ}$, and $2 \theta=34^{\circ}$ were attributed to the $\mathrm{t}$ $(111), \mathrm{t}(220)$, and $\mathrm{t}(002)$ planes of tetragonal phase ( $\mathrm{t}-$ ZrO2, JCPDS card No. 17-0923) of Zirconia, respectively [2, 24-28]. The results that obtained from our study are consistent with the results reported in previous studies. The peak intensity of the monoclinic and tetragonal phases were very weak when the Ar gas flow rate was the lowest. Furthermore, the peak intensity of $\mathrm{m}(-111)$ and $\mathrm{t}(111)$ has significantly increased with increasing $\mathrm{Ar}^{+}$partial pressure. Therefore, these results suggest that the crystal growth direction of zirconium oxide films depends on the Ar partial pressure.

The grain size (D) of the $\mathrm{ZrO}_{2}$ films was estimated by using Debye-Scherrer's Formula [29]
$D=\frac{K \lambda}{\beta \cos \theta}$,

where $\lambda$ is the wavelength of the $\mathrm{X}$-ray and $\beta$ the full width at half maximum (FWHM) of the $\mathrm{t}(111)$ peak of the XRD pattern. $K$ is the Scherrer constant which depends on the grain size geometry and size distribution. A value of 0.9 was used for K. Given the uncertainties in $K, D$ values calculated from the Scherrer equation should be considered as estimates only. As the Ar gas flow rate increases, the grain size also tends to increase, but the FWHM tends to decrease. Figure 2 shows the relationship between FWHM and gas flow rate. As the Ar gas flow rate increases, the particle size tends to decrease as shown in Fig. 2. Crystal structure and other characteristic properties of $\mathrm{ZrO}_{2}$ thin films are summarized in Table 1.

\subsection{AFM analyses}

The surface morphology of Zirconia thin films prepared on glass substrates at different Ar gas flow rates was examined by AFM. Figure 3 presents the AFM images of the zirconium oxide thin films in a three-dimensional format with a $5 \mu \mathrm{m} \times 5 \mu \mathrm{m}$ area. The film surfaces (Fig. 3.) are quite rough and cracks appear in some parts of the surfaces. The surface morphology of the zirconium oxide thin films changed significantly as the argon gas content increased. The grain size increased with increasing Ar gas flow

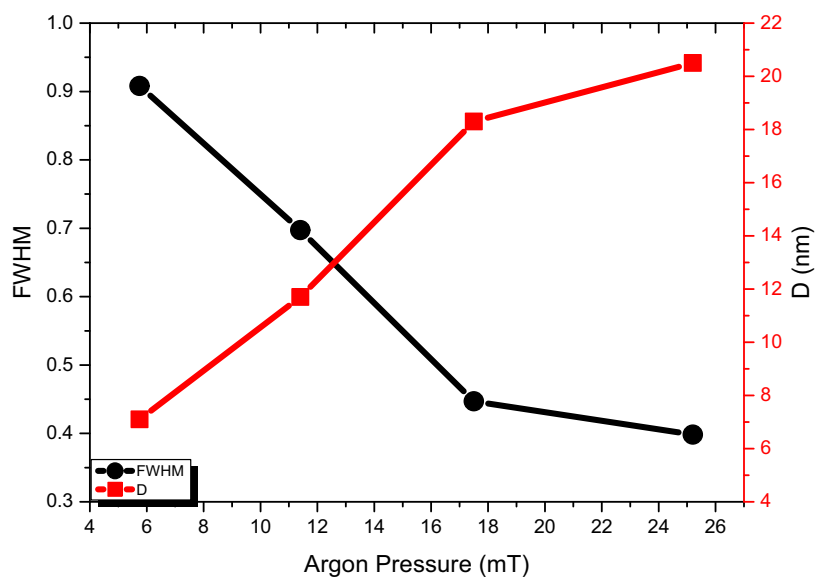

Fig. 2 Graphical presentation of FWHMs and particle sizes of Zirconium films produced using various Ar gas flow rates 
Table 1 Structural, morphological, and optical parameters of Zirconium thin films

\begin{tabular}{llllllll}
\hline Sample & Argon gas flow (sccm) & $(h k l)$ & $D(\mathrm{~nm})$ & FWHM (deg) & Rmax (nm) & $n($ at $550 \mathrm{~nm})$ & Eg (eV) \\
\hline ZO1 & 5 & $(220)$ & 7 & 0.908 & 42.5 & 1.76 & 3.26 \\
ZO2 & 10 & $(111)$ & 12 & 0.697 & 104.6 & 1.83 & 3.21 \\
ZO3 & 15 & $(111)$ & 18 & 0.447 & 217.5 & 1.94 & 3.21 \\
Z04 & 20 & $(111)$ & 20 & 0.398 & 18.3 & 2.12 & 3.16 \\
\hline
\end{tabular}
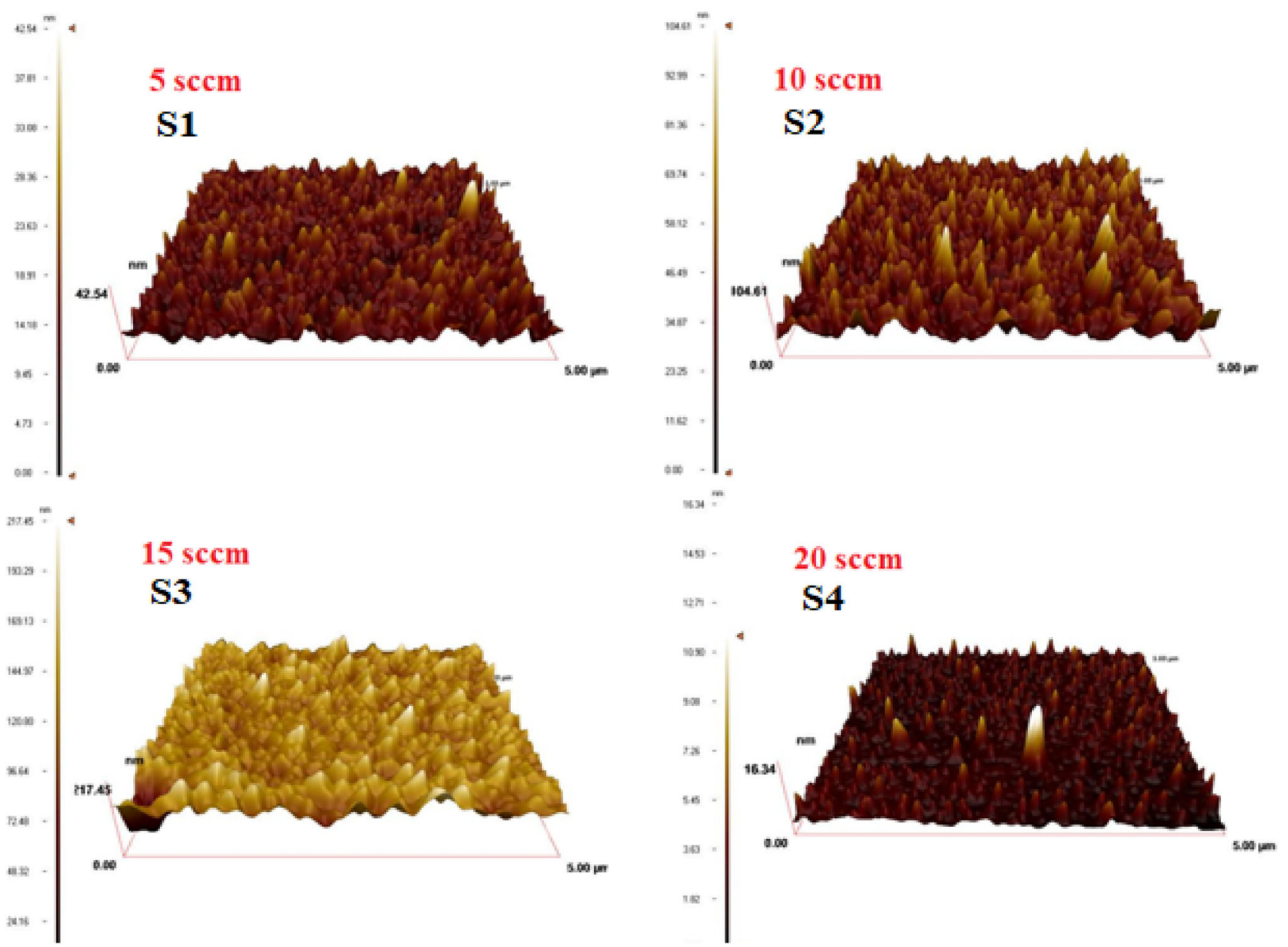

Fig. 3 AFM pictures $\left(\mathrm{S}_{1}, \mathrm{~S}_{2}, \mathrm{~S}_{3}, \mathrm{~S}_{4}\right)$ and surface roughness (e) for $\mathrm{ZrO}_{2}$ thin films with different argon gas flow rates

rates. Maximum peak height $\left(R_{\max }\right)$ values first tended to linearly increase from 42.5 to $217.5 \mathrm{~nm}$, then this roughness again decreased to $18.3 \mathrm{~nm}$. The minimum surface roughness at $20 \mathrm{sccm}$ is due to reduced boundaries between grains and surface evaporation of cage components [30,31]. It is a consensus that the increase of grain size decreases the film roughness obtained in the AFM measurements.
This is because of the fact that the roughness arises from the boundaries between the grains.

\subsection{SEM analyses}

The surface morphology and elemental stoichiometric ratios of Zirconia films produced in various Ar partial pressure environments using the Magnetron 
sputtering technique and their elemental stoichiometric ratios were determined by SEM and EDAX analyses (Fig. 4), respectively. It was determined that zirconium nanostructures produced using various Ar gas flow rates consist of almost homogeneous morphology and similar granular grains. SEM images show that the particle sizes of Zirconia nanostructures and the tightly packed structure of these particles are significantly altered by the Ar partial pressure. The size of the granules continuously increased with Ar partial pressure, and especially the granular nanostructure of S3 became more clear and uniform. SEM images also support the particle sizes of zirconium films detected using the XRD analyses. In addition, the $\mathrm{Zr}$ and $\mathrm{O}$ elemental composition of the films were determined by EDAX analysis. The stoichiometry of emission peaks such as $\mathrm{OK}$ and $\mathrm{ZrL}$ of the synthesized Zirconium nanoparticles was confirmed by the EDAX spectrum. The results obtained are similar to the existing studies in the literature [34]. However, this appears in cases of dominant orientation of oxide materials, depending on the surface orientation containing the oxygen or $\mathrm{Zr}$ component. XRD measurements indicate the dominant orientation as [111], resulting in low surface energy, except for $5 \mathrm{sccm}$ Ar gas flow rate. Low surface energy generally means low oxygen vacancies, resulting in high concentration of oxygen component.

\subsection{Optical studies}

Photoluminescence (PL) technique was used to determine the crystalline quality of the zirconium nanostructures and the presence of impurities.

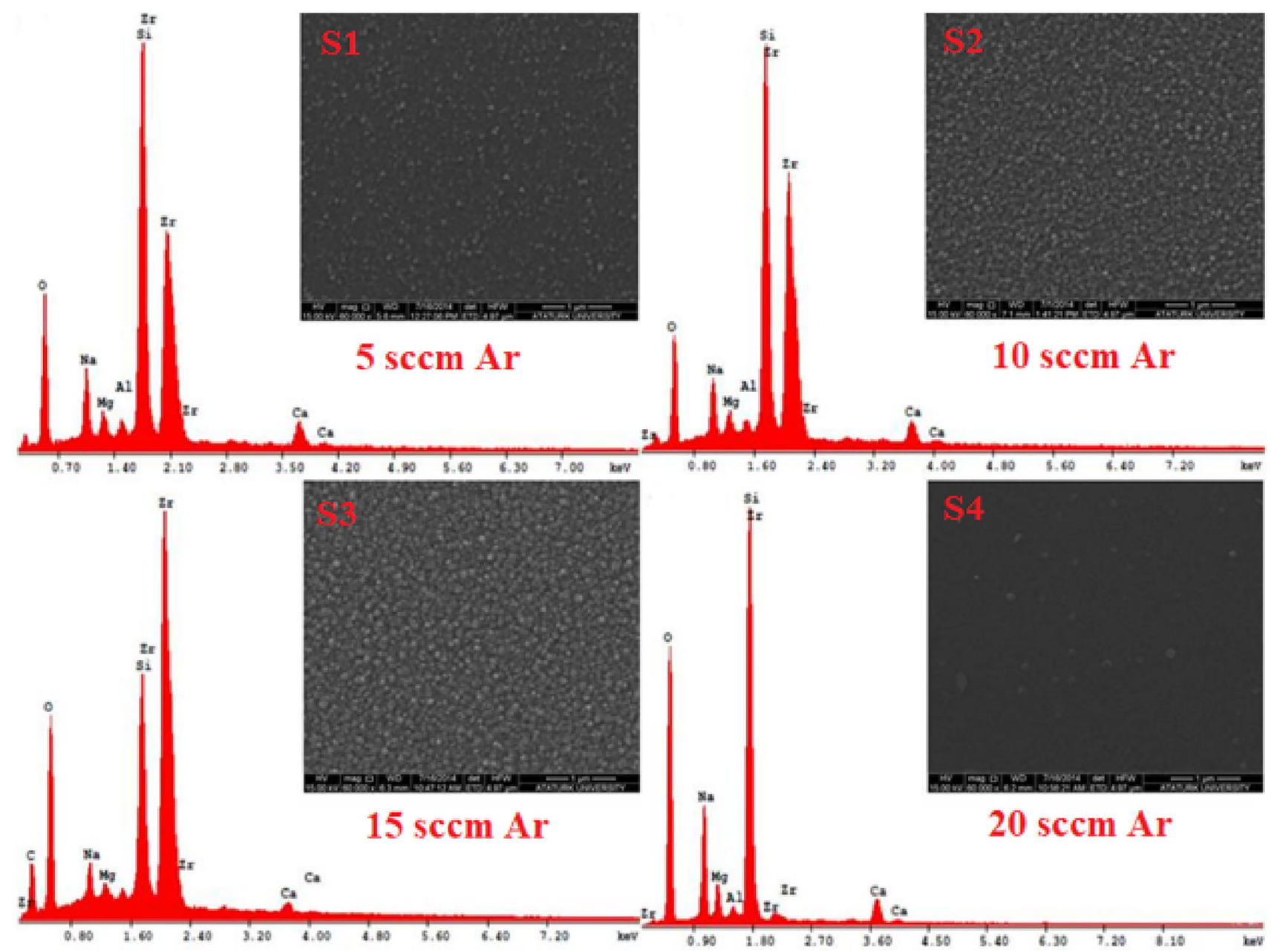

Fig. 4 SEM images and EDAX analysis of $\mathrm{ZrO}_{2}$ samples at different argon gas flow rates 
Figure 5 shows the spectrum of films deposited using various Ar partial pressures. The PL spectra of the Zirconium nanostructure deposited from the Ar partial pressure of S1 exhibit a significant near-bandedge emission peak at approximately $380 \mathrm{~nm}$, but no exciton peak is seen in any film. As the argon pressure increases, the formation of new peaks is observed in the emission peaks. These peaks are divided into two new peaks, as seen in Fig. 5, at 367 $\mathrm{nm}$ and $392 \mathrm{~nm}$. Apart from these peaks, no new peaks have been observed. As the Ar gas flow rates increased from $5 \mathrm{sccm}$ to $20 \mathrm{sccm}$, it shifted from 380 $\mathrm{nm}(3.27 \mathrm{eV})$ to $392 \mathrm{~nm}(3.13 \mathrm{eV})$, respectively. Analogous nature has been proposed for a broad emission observed from single crystals of Zirconia [32]. The characteristic absorption bands of $\mathrm{t}-\mathrm{ZrO}_{2}$ attributed to oxygen emission were partially observed in Zirconia [33, 34]. The most intense emission peak at $380 \mathrm{~nm}$ and can be due to the capture of electrons by oxygen vacancies from the conduction band [35]. Particle sizes detected using XRD analysis are the main cause of the shift in this spectrum of Zirconium thin films. Berlin et al. suggested that thin films are caused by a change in stress along the $c$ axis, depending on the particle size of the red shift in the PL spectrum [36]. Liang et al. revealed the existence of some weak peaks apart from these peaks in the study where they produced Zirconium thin films [37]. The absence of these weak peaks corresponding to the green luminescence in the PL analysis of thin films exhibiting a wide UV spectrum from 325 to about $500 \mathrm{~nm}$ is attributed to line expansion
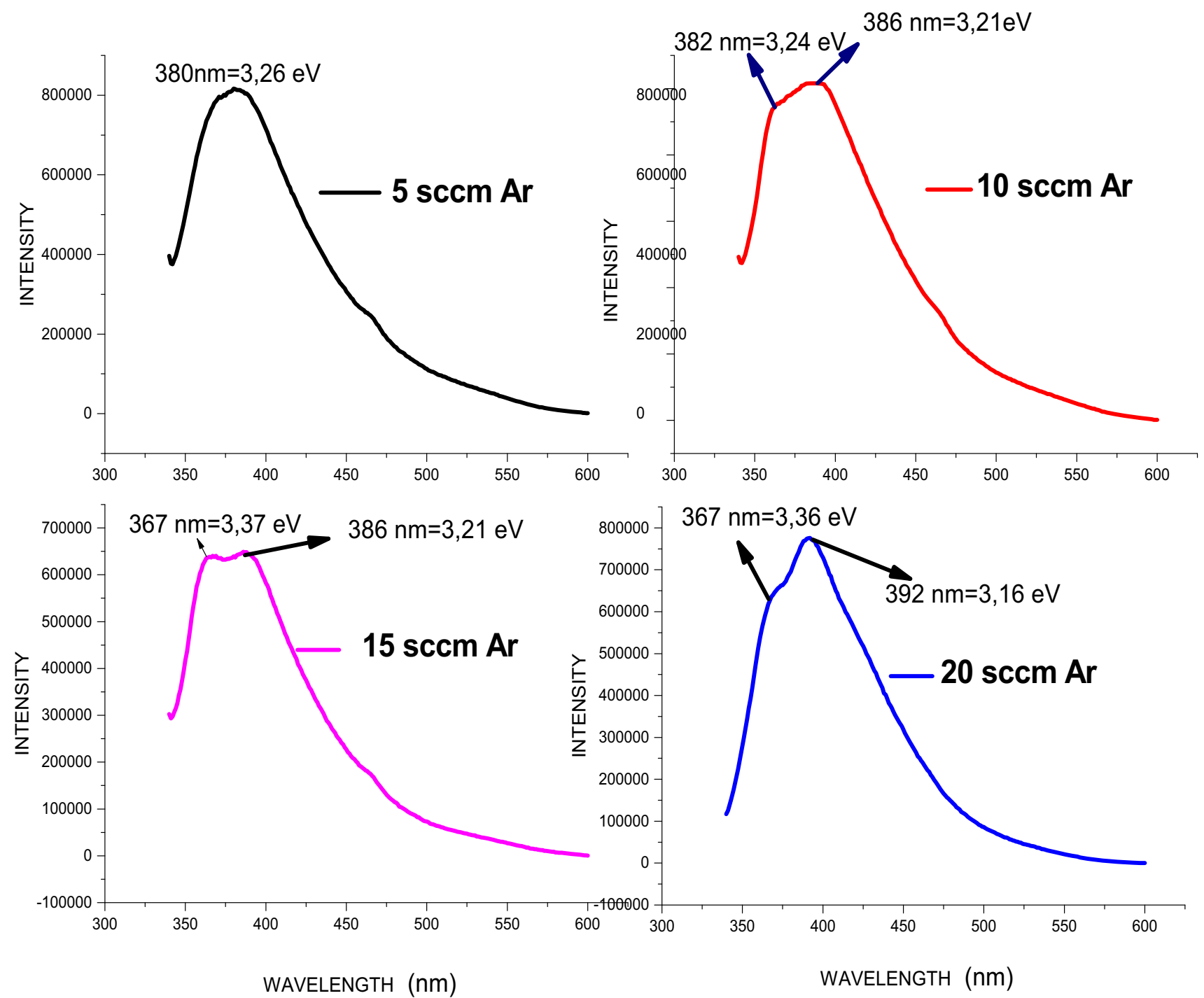

Fig. 5 PL spectra of Zirconia thin films produced at various Ar gas flow rates 
and overlaps. The excitation phase at $243 \mathrm{~nm}$, corresponding to the energy gap of the $\mathrm{ZrO}_{2}$ tetragonal phase and also attributed to grain boundary defect states, was not observed in any thin film.

Optical properties of thin films were also investigated using absorbance and transmittance analysis. Both absorbance and transmittance spectra were determined using Uv-Visible spectroscopy capable of measuring in the $200-900 \mathrm{~nm}$ range, and spectra as a function of the wavelength are presented in Figs. 6 and $7 \mathrm{~b}$, respectively. Figure 6 presents the absorbance plot of the S1-coded sample, which presents a sharp peak at approximately $310 \mathrm{~nm}$, attributed to surface

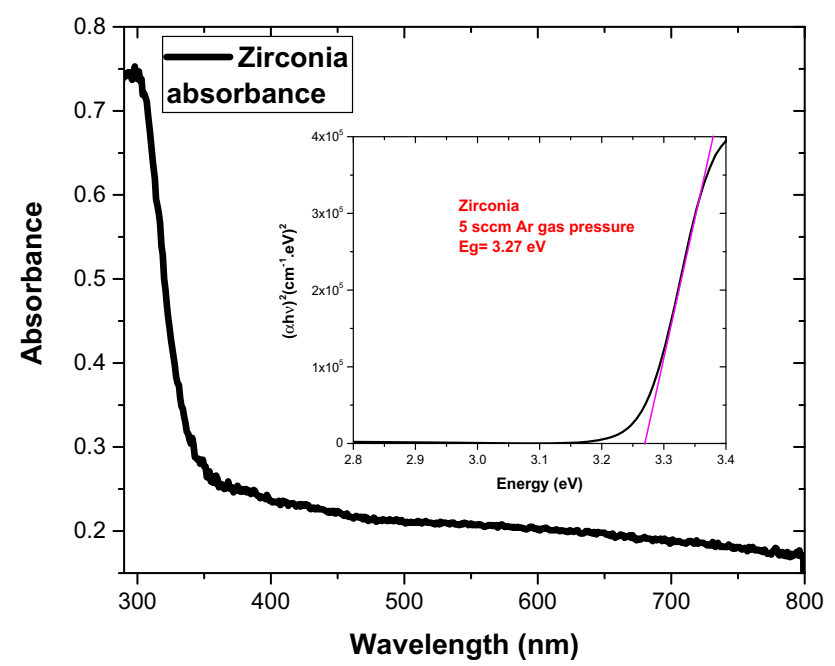

Fig. 6 Absorbance spectrum and Eg graps of S1

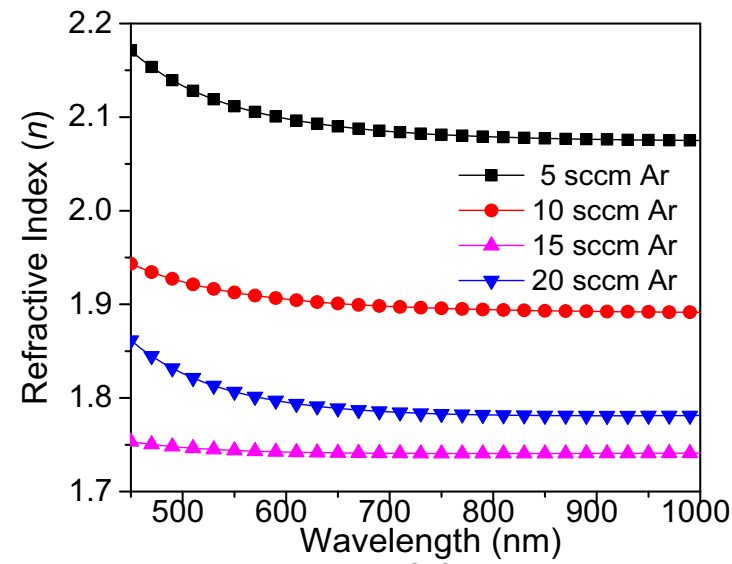

(a) defects and oxygen voids [38]. The absorption coefficient was calculated using the absorption spectrum presented in Fig. 6. The optical band gap of the S1coded thin film was calculated with the help of the Tauc relationship [39] and was correlated with the PL spectrum result. The optical band gap calculated using the Tauc equation was $3.27 \mathrm{eV}$, and this value is considered to be highly compatible with the $3.26 \mathrm{eV}$ determined for this film in the PL spectra. The optical transmittance of all thin films implies transparency for the apparent wavelength. It was determined that as the argon partial pressure increases for $550 \mathrm{~nm}$ wavelength, the optical transparency of the films increases. At this wavelength, the optical transparency of thin films has been improved from 70 to $95 \%$ with increasing partial pressure. In optical transmittance analysis, the formation of interference fringes, which is also attributed to the homogeneity and uniform morphology of the films, was observed [40]. Refractive index spectra determined using absorbance spectra were presented in Fig. 7a. The refractive indices of thin films were almost linear for the visible region wavelength. The refractive indices tended to increase slightly towards $300 \mathrm{~nm}$. As the partial pressure of argon increased, the refractive index decreased linearly, and decreased from 2.12 to 1.76 with increasing Ar partial pressure for $550 \mathrm{~nm}$ wavelength. This is because of the fact that the film refractive index is related to the film density and crystallization [24]. Some studies in the literature are also in good agreement with the refractive index values of zirconium thin films [41-43]. The high refractive index indicates that the prepared $\mathrm{ZrO}_{2}$

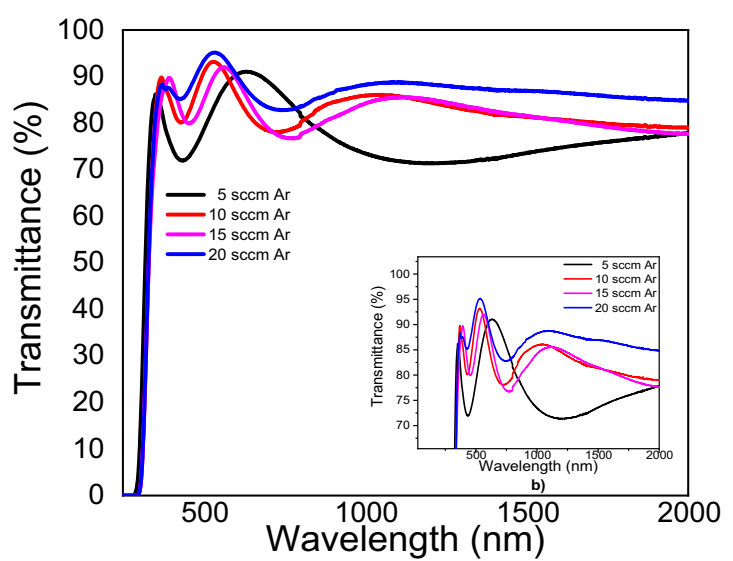

(b)

Fig. 7 The a refractive index and $\mathbf{b}$ transmittance spectra of $\mathrm{ZrO}_{2}$ thin films on glass substrates 
films can be a potential optical coating material. When the transmission measurements are examined, all the films exhibit a high transmittance $(\sim 95 \%)$ in the visible region, and therefore, $\mathrm{ZrO}_{2}$ films are a very good candidate materials for transparent electronic devices because of their high transmittance.

\section{Conclusion}

Zirconia thin films were successfully grown for various Ar gas flow rates using the RF magnetron scattering technique. For all thin films, it was found that gas pressure had an effect on film quality. Surface morphologies were significantly affected by this gas flow, but this effect was not linear. The optical analysis of thin films showed that the films were transparent and this value was approximately $95 \%$ at 550 nm wavelength. In addition, the optical band gap of the films was calculated by PL analysis, the thin films had a band gap of about $3.25 \mathrm{eV}$. Ar gas flow rate did not significantly affect this value. Furthermore, the dielectric characteristics were calculated between 1.76 and 2.12 at $550 \mathrm{~nm}$ wavelength. All morphological and structural properties of the films showed that they had the potential to be used in the TCO industry.

\section{Author contributions}

PKB, EG, and OB conducted the experiments, performed the computations, and verified the analytical methods. OB supervised the findings of this work. All authors discussed the results and contributed to the final manuscript.

\section{Compliance with ethical standards}

Conflict of interest The authors declare that they have no known competing financial interests or personal relationships that could have appeared to influence the work reported in this paper. The authors declare the following financial interests/ personal relationships which may be considered as potential competing interests: Pinar K. Bakacak, Emre Gur, Ozkan Bayram, Sebahattin Tuzemen, and Onder Simsek.

\section{References}

1. J.H. Ko et al., Characteristics of $\mathrm{ZrO}_{2}$ thin films deposited by reactive magnetron sputtering. J. Korean Phys. Soc. 50, 1843 (2007)

2. J. Park, J.K. Heo, Y.-C. Kang, The properties of RF sputtered zirconium oxide thin films at different plasma gas ratio. Bull. Korean Chem. Soc. 31(2), 397 (2010)

3. A. Singh, U.T. Nakate, Microwave synthesis, characterization, and photoluminescence properties of nanocrystalline zirconia. Sci. World J. (2014). https://doi.org/10.1155/2014/ 349457

4. S. Venkataraj et al., Structural and optical properties of thin zirconium oxide films prepared by reactive direct current magnetron sputtering. J. Appl. Phys. 92(7), 3599-3607 (2002)

5. L.-J. Lai et al., Photoluminescence of zirconia films with VUV excitation. J. Electron Spectrosc. Relat. Phenom. 144, 865-868 (2005)

6. J. Gottmann et al., Optical properties of alumina and zirconia thin films grown by pulsed laser deposition. Surf. Coat. Technol. 100, 415-419 (1998)

7. M.N. Tahir et al., Facile synthesis and characterization of monocrystalline cubic $\mathrm{ZrO}_{2}$ nanoparticles. Solid State Sci. 9 (12), 1105-1109 (2007)

8. M. Huang et al., $\mathrm{ZnO}$ microrods photodeposited with $\mathrm{Au}-\mathrm{Ag}$ nanoparticles: synthesis, characterization and application. Sers. Sci. 292, 1897 (2001)

9. S. Park, J.M. Vohs, R.J. Gorte, Direct oxidation of hydrocarbons in a solid-oxide fuel cell. Nature 404(6775), 265 (2000)

10. X.X. Feng, L.R. Fang, ZHENG Yang Zeng ( $\square \square)$. Chin. J. Inorg. Chem.(Wuji Huaxue Xuebao) 21(7), 965-970 (2005)

11. C.-Y. Lin et al., Effect of top electrode material on resistive switching properties of $\mathrm{ZrO}_{2}$ \&nbsp;film memory devices. IEEE Electron. Device Lett. 28(5), 366-368 (2007)

12. J. Koo, Y. Kim, H. Jeon, $\mathrm{ZrO}_{2}$ gate dielectric deposited by plasma-enhanced atomic layer deposition method. Jpn. J. Appl. Phys. 41(5R), 3043 (2002)

13. H. Suematsu et al., Preparation of $\mathrm{Zr}-\mathrm{Si}$ thin films by a simultaneous deposition and reaction process using pulsed ion beams. Mater. Lett. 61(17), 3635-3638 (2007)

14. A.M. Torres-Huerta et al., MOCVD of zirconium oxide thin films: synthesis and characterization. Appl. Surf. Sci. 255(9), 4792-4795 (2009)

15. Y. Komatsu et al., Preparation of $\mathrm{YBCO} / \mathrm{ZrO}_{2}$ thin films on $\mathrm{Si}$ by MOCVD using a mode converting type of microwave plasma apparatus. Thin Solid Films 341(1-2), 132-135 (1999) 
16. O. Bayram et al., Effect of the number of cycles on the optical and structural properties of $\mathrm{Mn}_{3} \mathrm{O}_{4}$ nanostructures obtained by SILAR technique. J. Mater. Sci. Mater. Electron. 29(12), 10542-10549 (2018)

17. O. Bayram et al., The role of cobalt doping on the optical and structural properties of $\mathrm{Mn}_{3} \mathrm{O}_{4}$ nanostructured thin films obtained by SILAR technique. Superlattices Microstruct. 128, 212-220 (2019)

18. O. Bayram et al., Effect of doping concentration on the structural and optical properties of nanostructured $\mathrm{Cu}$-doped $\mathrm{Mn}_{3} \mathrm{O}_{4}$ films obtained by SILAR technique. Appl. Phys. A 124(9), 606 (2018)

19. O. Bayram et al., Synthesis and characterization of Zn-doped $\mathrm{Mn}_{3} \mathrm{O}_{4}$ thin films using successive ionic layer adsorption and reaction technique: Its structural, optical and wettability properties. J. Mater. Sci. Mater. Electron. 29, 9466-9473 (2018)

20. S. Venkataraj et al., Thermal stability of sputtered zirconium oxide films. Vacuum 75(1), 7-16 (2004)

21. Z. Ji et al., Metastable tetragonal zirconia formation and transformation in reactively sputter deposited zirconia coatings. Surf. Coat. Technol. 135(2-3), 109-117 (2001)

22. O. Bayram, Conjugated polythiophene/Ni doped $\mathrm{ZnO}$ hetero bilayer nanocomposite thin films: its structural, optical and photoluminescence properties. Ceram. Int. 44(17), 2063520640 (2018)

23. O. Bayram et al., Investigation of structural, morphological and optical properties of Nickel-doped Zinc oxide thin films fabricated by co-sputtering. J. Mater. Sci. Mater. Electron. 30 (4), 3452-3458 (2019)

24. L. Zhu et al., Interfacial and optical properties of $\mathrm{ZrO}_{2} / \mathrm{Si}$ by reactive magnetron sputtering. Mater. Lett. 60(7), 888-891 (2006)

25. X. Jiao, D. Chen, L. Xiao, Effects of organic additives on hydrothermal zirconia nanocrystallites. J. Cryst. Growth 258 (1-2), 158-162 (2003)

26. S. Jayakumar et al., Nanosize stabilization of cubic and tetragonal phases in reactive plasma synthesized zirconia powders. Mater. Chem. Phys. 140(1), 176-182 (2013)

27. Y. Zhou et al., Thickness dependence of the structural and dielectric properties of epitaxial $\mathrm{ZrO}_{2}$ films grown by limited reaction sputtering. J. Phys. D Appl. Phys. 42(20), 205406 (2009)

28. L. Kumari et al., Controlled hydrothermal synthesis of zirconium oxide nanostructures and their optical properties. Cryst. Growth Design 9(9), 3874-3880 (2009)

29. P Köç et al., Stimulated electroluminescence emission from $\mathrm{n}-\mathrm{ZnO} / \mathrm{p}-\mathrm{GaAs}$ : $\mathrm{Zn}$ heterojunctions fabricated by electro-deposition. AIP Adv. 3(12), 122107 (2013)
30. D Kohl, M Henzler, G Heiland, Low temperature sublimation processes from clean cleaved polar surfaces of zinc oxide crystals during first heating. Surf. Sci. 41(2), 403-411 (1974)

31. C.V. Thompson, Structure evolution during processing of polycrystalline films. Annu. Rev. Mater. Sci. 30(1), 159-190 (2000)

32. S.K. Batygov et al., Self-localized elementary excitations in stabilized zirconium dioxide crystals. Sov. Phys. Solid State 30, 378-380 (1988)

33. V. Strekalovsky, Y.M. Polejaev, S. Palguev, Oxides with Extrinsic Disorder (Nauka, Moscow, 1987).

34. V. Kiisk et al., Photoluminescence of sol-gel-prepared hafnia. Phys. B 405(2), 758-762 (2010)

35. A.S. Foster et al., Structure and electrical levels of point defects in monoclinic zirconia. Phys. Rev. B 64(22), 224108 (2001)

36. I.J. Berlin et al., Enhancement of photoluminescence emission intensity of zirconia thin films via aluminum doping for the application of solid state lighting in light emitting diode. J. Lumin. 132(11), 3077-3081 (2012)

37. J. Liang et al., Characterization and synthesis of pure $\mathrm{ZrO}_{2}$ nanopowders via sonochemical method. Mater. Res. Bull. 38 (1), 161-168 (2003)

38. B. Sathyaseelan et al., Studies on structural and optical properties of $\mathrm{ZrO}_{2}$ nanopowder for opto-electronic applications. J. Alloy. Compd. 694, 556-559 (2017)

39. J. Tauc, Amorphous and Liquid Semiconductors (Plenum, London, 1974).

40. A. Hojabri, Structural and optical characterization of $\mathrm{ZrO}_{2}$ thin films grown on silicon and quartz substrates. J. Theor. Appl. Phys. 10(3), 219-224 (2016)

41. Y. Shen et al., Influences of oxygen partial pressure on structure and related properties of $\mathrm{ZrO}_{2}$ thin films prepared by electron beam evaporation deposition. Appl. Surf. Sci. 254 (2), 552-556 (2007)

42. F. Rebib et al., Investigation of structural and optical properties of sputtered Zirconia thin films. Eur. Phys. J. Appl. Phys. 43(3), 363-368 (2008)

43. K. Joy et al., Effects of annealing temperature on the structural and photoluminescence properties of nanocrystalline $\mathrm{ZrO}_{2}$ thin films prepared by sol-gel route. J. Phys. Chem. Solids 72(6), 673-677 (2011)

Publisher's Note Springer Nature remains neutral with regard to jurisdictional claims in published maps and institutional affiliations. 